\title{
Question of Color Perception in Literary Work (Based on Boris Pasternak's Doctor Zhivago)
}

\author{
Yulia Dyupina*, and Tatiana Shakirova \\ Tyumen Industrial University, Department of Interpersonal Communications, 625000, Tyumen, Russia
}

\begin{abstract}
This work demonstrates the experiment on the perception and generation of the text as a part of the literary text analysis. The author was faced with a task to estimate the ability of participation of color terms in formation of art sense of the text as a whole. The emphasis is placed on the identification of expansion of color terms in their semantics when they go beyond the designation of color itself and become multidimensional and multiple-valued, taking root in such difficult structures as a metaphor, a comparison, etc. The cases of discrepancy between the choice of color words by recipients (participants of the experiment) and an author's conception of color definitions are analyzed. The exclusiveness of an author's colors seeing is emphasized.
\end{abstract}

The names of color function actively in language. Quite naturally, the word-color terms have long attracted the attention of philologists. First of all, researchers are trying to answer the question, what is the significance of the concept "red", "black", "white", etc. [1-3] In other words, scientists are interested in how the color categories are reflected in human consciousness. It is known that the value of each culture in the same color terms is different. As noted by Brent Berlin and Paul Kay, in the Maori language (indigenous people of New Zealand) there are more than a hundred shades of color, which is usually defined as "red". [4] Another example the English blue in the Russian language corresponds to "синий" [blue] and "голубой" [light blue], it means that native English and Russian language carriers in this case speak of different colors which, however, does not disturb the process of communication in translation.

Behind words-color terms in different languages there is an extensive information potential which represents a complex multilevel color symbolism. Throughout the history people studied the different symbols which helped them distinguish and understand any phenomenon. These symbols warned, directed and informed a man in different areas of knowledge. The colors have been used in symbolism since the ancient times. The color values and the associations they cause are different in different cultures. [5] Guinness World Publishing House prepared and published "The Etymology of Signs and Symbols" which presents a variety of characters, from the simplest to the most complex, including color.

Therefore, the problem of existence and functioning of words in the language of color terms and consciousness cannot be limited by only linguistic or literary studies. The attempts to determine the nature of color are taken in psychology and philosophy and in other related fields. [6,7] It is obvious to assume that even with the native language "intuition" of one person (including a linguistic specialist) cannot always serve as the standard which is truly reflecting the collective linguistic ethnical consciousness of native speakers.

Creating the art text, the author makes a selection of those phenomena of reality which correspond to his representations or concepts. [8] Fictional, but plausible situations are modeled by the author for the explanation and confirmation of the ideas and representations.

The interpretation of the text assumes the existence of certain skills of interpretation as specific actions with contents of the text, peculiar "rules" and "strategy" of interpretations. They represent a special type of knowledge which is stored in memory of a man as an action program with a certain object text at a meeting with him in the framework of an activity. As well as any knowledge, "rules" and "strategy" of interpretation have to be acquired by the reader. This formation can occur spontaneously and perhaps unnoticed by a man in circumstances where he is involved in certain activities because of certain circumstances of life, like an object, which determines the production and mastering the rules of action with this object.

On the conscious and subconscious levels of a man the associations and concepts are born associated with certain colors. This genetically solidified many centuries of human evolution in its collective unconscious. [9] Since the childhood a man in the process of acquisition of life experience has a representation how this or that color looks. For example, red is traditionally associated with the color of blood, blue - the color of sky, green the color of grass. It should be noted that the basis for retrieval from memory during the reading and generation of the text is the detection of similarity between the readable text and some texts which were familiar earlier.

* Corresponding author: ta-nusha9@mail.ru 
It is necessary to emphasize that the color terms find the ability to participate in formation of art sense of the whole text. In the structure of text the color terms join in an author's and personages' composite and speech segments, in "strong positions" of the text, can be the elements of steady formulas of a narrative or graphic exposition of the character, i.e. possess inexhaustible opportunities in expression of a boundless variety of values and meanings, in the image of the widest ranges of human thoughts, feelings and aspirations. Color terms can be used in their core values as the color characteristics, as well as to go beyond the actual color designations, undergo a kind of evolution from the simple designation of color to express feelings, inner world of a character, its mood and even abstract ideas that cannot be represented visually.

The ability of the color terms to transfer an emotional state, to express feelings can be explained as from linguistic and non-linguistic positions. It is known that a human being is emotional by the nature. A man and his emotions, on the one hand is the part of the objective reality, but on the other is involved in the formation of linguistic world. At the same time the knowledge of surrounding reality is carried out and due to color sight. Moreover, the emergence of the color terms in the language (as previously mentioned in this study) has the character combinations / comparisons with color shades of events and objects that are already known to native speakers, i.e. "meaning" of color terms arise by virtue of their relationship to human experience.

One of the successful methods of knowledge can be a linguistic experiment on the perception and the generation of the text as a part of the analysis of the text. The assessment of semantics, forms and functions of the units making an art work is formed and constantly develops in our minds at perception and generation of the text. [10]

When carrying out the experiment, we were interested whether the way of perception and generation of the text is universal or each of them proceeds differently. The first part of the experiment was to fill the gaps in the sentence. The 1-3 year students of the Tyumen State Architectural and Engineering University (116 people) acted as recipients. Their task consisted in filling the gaps in the sentences with semantics of color. The fragment from Boris Pasternak's "Doctor Zhivago" was offered. Its original form was as follows:

The young forest under an embankment was almost empty as in the winter. Only the buds in which it was completely dripped like wax looked like something extra, some disorder, such as dirt or swelling, and this odd, these irregularities and dirt was life, green flame foliage coverage of the first blossoming trees in the forest.

(Boris Pasternak, Doctor Zhivago).

The following option was offered to the examinees:

The young forest under an embankment was almost empty as in the winter. Only the buds in which it was completely dripped like wax looked like something extra, some disorder, such as dirt or swelling, and this odd, these irregularities and dirt was life, flame foliage coverage of the first blossoming trees in the forest.

The students needed to pick up the color definition relating to flame foliage.

The lexeme choice of green is justified since the author shows the process of changing seasons, the coming of spring. The words the young forest only underline the emergence of new life in the nature. Green color is traditionally assigned to color of leaves and trees. Most students chose a color term green and its shades $(52 \%)$. However the lexical environment (dirt, swelling, dripped like wax) provoked the use of color terms of other color group, for example, brown, dark brown, black, etc. $(4 \%)$. It is interesting that the choice of color terms by some recipients was affected by the metaphor foliage flame, some students offered the following color terms: red $(9 \%)$, bright red $(6 \%)$, bright yellow (3\%). The mismatch with the author's intention of red color is clearly linked to the image of fire in this passage.

Traditionally the color of clear sky, water, rain, snow and ice communicates with white, shades of blue and light blue. The author of "Doctor Zhivago" often draws the winter landscapes; however one of distinctive features in a color picture of Pasternak's world is the special feelings to the color of a winter landscape usually it is dark, even black disturbing colors. In the following fragments offered to recipients, the author's value of shades of dark color is just realized:

Only now, for the second time having gone outside, Lara plainly looked around. There was a winter. There was an evening. There was icy cold. Streets were covered by black ice, thick like glass bottoms of beaten beer bottles. It hurt to breathe.

There was a dry frosty day of the beginning of November, with a lead-gray quiet sky and few snowflakes which long and evasively hung before falling to the ground and then to be hammered with gray fluffy dust into road potholes.

(Boris Pasternak, Doctor Zhivago).

The ice was characterized by most of examinees as transparent $(32 \%)$ that corresponds to dictionary value of this word. Also there were such definitions named as white $(11 \%)$, gray $(9 \%)$, light blue $(5 \%)$, silvery $(5 \%)$, and blue $(2 \%)$. These color definitions are traditionally assigned to ice, and this choice by the recipients, in our opinion, is reasonable. For the same reasons any of examinees could not recreate the author's option of a color term (black ice) that only proves the exclusiveness of the author's color vision in some cases. However, some attempts to take the following scheme for a reference point should be noted:

winter $\rightarrow$ evening $\rightarrow$ evening city $\rightarrow$ dark evening streets $\rightarrow$ black sidewalks $\rightarrow$ dark ice

As a result some students chose the colors dark (1\%) and dark blue $(0.5 \%)$.

Another option of winter pictures - the quiet sky is painted in a dry frosty day. The lexical accompaniment caused a choice of color words by the recipients: light blue $(24 \%)$, blue $(35 \%)$ and their shades $(21 \%)$. This time nobody could reproduce the author's color term lead-gray and that is quite predictable, first of all, 
because lead-gray sky is the author's epithet, and you have to be Pasternak to call the clear frosty sky leadgray; secondly, in the fragment there are no lexical prerequisites for the use of the color term with such saturated dark shade.

"Doctor Zhivago" is a good material for the research in the field of studying features of the use of color terms. The rich color palette, non-standard color oppositions, new symbolical values of colors - Pasternak shows the abilities of the true literary artist. One of his features of the color choice is that the author seldom uses a single color term. Usually the shades of one color supplement each other, the color image thereby turns out especially rich and exact. One of such images was offered to recipients:

She held a bone mouthpiece with a cigarette in the yellowed teeth, dropping an eye with the yellow white and releasing a yellow stream of a smoke with mouth and nose, wrote down in a notebook the measurements, numbers of receipts, addresses and wishes of the crowding customers.

(Boris Pasternak, Doctor Zhivago).

Students were asked to restore the unit with semantics of color relating to a smoke stream. The intentional concentration of lexemes of yellow color in the description of the heroine is evident, nevertheless, many of students did not see yellow and chose a lexeme of gray color $(29 \%)$ and its shades: muddy-gray, dark gray, whitish-gray, gray-ash, matte-gray, dim gray, pale gray, etc. $(17 \%)$, being guided, probably, by the traditional color ideas of a smoke. However, some of examinees showed the language insight and chose the color yellow (9\%) and its shades: yellowish, poisonousyellow, muddy-yellow (2\%).

When choosing the color definitions for these words and phrases regardless of coincidence or discrepancy to the author's option the student audience surprised with a big variety of color schemes. First of all it concerns the spread of color shades, sometimes unexpected and even inconsistent (dark-white, silver-diamond). The analysis of the material showed that the students actively used sophisticated color terms formed within one color (muddy-yellow, pale gray, light pink), and different ones (blue-white, silvery-violet). Noteworthy are the definitions given by the students which represent a combination of two types of perception: color and tactile (soft-blue, silky-ashy).

The second part of the experiment represented a free reproduction of experimental words. The examinees were offered a number of comparative structures in which the object of comparison was omitted. The following comparative structures were offered to the students:

1)The weather was the most awful what can only be thought up. The biting gusty wind carried the torn shreds of clouds low to the ground, black like flakes of the flying soot. Suddenly from them it started snowing in convulsive haste of some white insanity.

2)With the heart pounding Lara walked through the empty streets. On the way, there were teahouses and taverns with doors smoking. From the fog came up the frosted faces of passers-by, red like sausage, and bearded muzzles of horses and dogs in icicles.

3)She often sent for Yura and Tonya and for hours told them about her childhood carried out in a grandfather's manor Varykina near the Ural river Rynva. Yura and Tonya have never been there, but Yura listening to Anna Ivanovna easily imagined these five thousand tithe of age-old impassable wood black like night where in two or three places the fast river with a rocky bottom and a high steep of the Kruger shore pierces like a knife with its bends.

4)Long ago the winter came. There were bitter colds. The broken-off sounds and shapes without visible connection appeared in the frosty mist, standing, moving, disappearing. Not the sun we are used to on the earth but some other, changed, a crimson sphere hung in the wood. The rays of thick amber-yellow like honey light, hardly and slowly like in a dream or in a fairy tale, were spreading in the air and freezing to the trees.

(Boris Pasternak, Doctor Zhivago).

Let us analyze each of the examples in terms of the correlation of the associative reactions with the semantic structure of the vocabulary of native speakers.

In the first example, the element of comparison was omitted and the text was as follows:

The weather was the most awful what can only be thought up. The biting gusty wind carried the torn shreds of clouds low to the ground, black like Suddenly from them it started snowing in convulsive haste of some white insanity.

The author as an object of comparison chose the image of flakes of the flying soot. It should be noted that the opinion of $18 \%$ of examinees almost coincided with the author's: they used smoke-black and ashes as an object of comparison which are very close in a lexical meaning; one student brought an object - the scorched flakes of papers which is also close to the author's option.

Being guided by the knowledge and life experience or the mood at the time of perception, the examinees compared the torn shreds of clouds with night $(22 \%)$, coal $(15 \%)$, smoke $(4 \%)$, dirt $(3 \%)$.

In the second example, we omitted the object which is associated with the faces of passers-by:

With the heart pounding Lara walked through the empty streets. On the way, there were teahouses and taverns with doors smoking. From the fog came up the frosted faces of passers-by, red like bearded muzzles of horses and dogs in icicles.

It is interesting that the majority of respondents (62\%) chose the tomato as an object of comparison. In our opinion such unanimity is caused by the stable unit of language - red like tomato. As expected, none of the examinees could offer the author's option - sausage. However, many students having shown their imagination chose even more exotic objects of comparison: strawberry, radish, carrot, mountain ash, comb of a rooster, rose, etc.

In the third case when it was necessary to call the object of comparison in relation to wood, the text had the following appearance: 
She often sent for Yura and Tonya and for hours told them about her childhood carried out in a grandfather's manor Varykina near the Ural river Rynva. Yura and Tonya have never been there, but Yura listening to Anna Ivanovna easily imagined these five thousand tithe of age-old impassable wood black like where in two or three places the fast river with a rocky bottom and a high steep of the Kruger shore pierces like a knife with its bends.

The options of the offered object have a wide spreading: cave $(4 \%)$, resin $(4 \%)$, cat $(4 \%)$, raven $(1 \%)$, coal $(6 \%)$. These objects are placed in one row because they do not bear, in our opinion, any additional information except color. With attentive reading of this structure, it is possible to track the expressiveness degree of the author's understanding (if well developed figurative and associative thinking prevails). In this particular case, the environment of words and phrases helps to reveal the psychological informational content: age-old, impassable wood. It caused the associations with haze (9\%), darkness $(7 \%)$, abyss (4\%). These text units bear the psychological information: an eternity, a gloom plus a lexeme black (wood) give the association with night. It is indicative that a choice of author's option is on the first place among the options of respondents, the total of coincidence in this case made $58 \%$.

The fourth example looked as follows:

Long ago the winter came. There were bitter colds. The broken-off sounds and shapes without visible connection appeared in the frosty mist, standing, moving, disappearing. Not the sun we are used to on the earth but some other, changed, a crimson sphere hung in the wood. The rays of thick amber-yellow like light, hardly and slowly like in a dream or in a fairy tale, were spreading in the air and freezing to the trees.

Among the named objects were jam (2\%), gold (2\%), fog $(13 \%)$, jelly $(10 \%)$, liqueur $(4 \%)$, caramel $(5 \%)$. The coincidence to the author's option made $29 \%$. In this example, all elements of a comparative structure could have impact on the recipient: first, rays were spreading (it indicates the viscosity of an object), secondly, thick (it indicates an object's consistence) and, at last, the direct indication of the color - amber-yellow. All specified signs are successfully combined in the author's option and allow making the right choice of the object of comparison. Despite it, many students considered the viscosity and consistence (sour cream, condensed milk, jelly, caramel, liqueur, jam, etc.), or only color (the sun, a rye field, sunshine, etc.). However the choice as an object of comparison of resin $(8 \%)$ and melted gold $(15 \%)$, in our opinion, coincides with the author's idea since they bear the same information.

The coincidence percent, as it seems to us, depended on the felicity of a verbal embodiment of the text content, on its style and on the interest degree of the reader.

Thus, during the experiment it was established that the influence of text units with semantics of color on the reader depends on the interest degree of the reader; on the expressiveness degree of the author's understanding; on the felicity of a verbal embodiment of the main contents of the text, on its style; on the development of figurative and associative thinking of the reader; on the psycho-emotional condition of the reader at the time of perception of the text. The impact of the text on any reader will be that more, than more convincingly it represents the conditions and ideas inherent in this type of mind. The lexicon with a value of color is one of the most important tools for verbal picturesqueness and art figurativeness. This lexicon allows the literary artist to show the depicted in a direct presentation, "the seeing". The words with a value of color realize in the text, first of all, the esthetic opportunities which at the lexical level are shown in variety of semantic links between words.

This sort of experiments has the important practical value. The offered tasks allow students to develop and improve the skills of the linguistic analysis of the text. The developed technique teaches them to see the text, develops the figurative and associative thinking. The experiment of generation and perception of the text unit with semantics of color confirmed the assumption that the perception of color also reflects the individual idea which can coincide or not coincide with the standard. All this is reflected in art texts of Boris Pasternak, during the experiment the objectivity of text information, the reality of their semantic importance for native speakers and for the Russian culture is defined.

\section{References}

1. R. Osborne, Colour Design 507-527 (2017)

2. A.J. Elliot, M.A. Maier, Advances in experimental social psychology 45, (2012)

3. B. Mikellides, Colour design 193-214 (2017)

4. B. Berlin, P. Kay, Basic Color Terms: Their Universality and Evolution (Los Angeles; Berkeley, 1969)

5. R. Frumkina, Language and Thinking as the Problem of Linguistic Experiment (News of the Academy of Sciences of the USSR. Literature and Language Series 3, 1981)

6. C.L. Hardin, Azimuth 1, 209-219 (1998)

7. R. McKimm, Colour design 215-239 (2017)

8. V. Kulpina, Linguistics Color (Moscow: Moscow Lyceum, 2001)

9. R. Frumkina, Color, Sense, Similarity: The Aspects of the Psycholinguistic Analysis (Moscow, 1984)

10. Z. Tarlanov, Methods and Principles of the Linguistic Analysis (Petrozavodsk, 1988) 